PROCEEDINGS OF THE

AMERICAN MATHEMATICAL SOCIETY

Volume 127, Number 3, March 1999, Pages 835-845

S 0002-9939(99)04751-6

\title{
FINITE FAMILIES WITH FEW SYMMETRIC DIFFERENCES
}

\author{
ALBERTO MARCONE, FRANCO PARLAMENTO, AND ALBERTO POLICRITI
}

(Communicated by Andreas R. Blass)

\begin{abstract}
We show that $2^{\left\lceil\log _{2}(m)\right\rceil}$ is the least number of symmetric differences that a family of $m$ sets can produce. Furthermore we give two characterizations of the set-theoretic structure of the families for which that lower bound is actually attained.
\end{abstract}

\section{INTRODUCTION}

Throughout this paper $\mathbf{F}$ and $\mathbf{G}$ will always denote finite families of sets.

Definition 1.1. - Let $A \Delta B$ denote the symmetric difference between the sets $A$ and $B$, defined as

$$
A \Delta B=\{x \mid(x \in A \wedge x \notin B) \vee(x \notin A \wedge x \in B)\} .
$$

- For $\mathbf{F}$ and $\mathbf{G}$ families of sets and $A$ a set let

$$
\begin{aligned}
\Delta \mathbf{F} & =\{A \Delta B \mid A, B \in \mathbf{F}\} ; \\
\bar{\Delta} \mathbf{F} & =\text { the closure under } \Delta \text { of } \mathbf{F} ; \\
\mathbf{F} \Delta \mathbf{G} & =\{A \Delta B \mid A \in \mathbf{F} \wedge B \in \mathbf{G}\} ; \\
A \Delta \mathbf{F} & =\{A \Delta B \mid B \in \mathbf{F}\} ; \\
A \cap \mathbf{F} & =\{A \cap B \mid B \in \mathbf{F}\} .
\end{aligned}
$$

Notice that $\emptyset \in \Delta \mathbf{F}$ for any $\mathbf{F}$, while $\emptyset \in \mathbf{F} \Delta \mathbf{G}$ if and only if $\mathbf{F} \cap \mathbf{G} \neq \emptyset$.

If $B \neq C$, then $A \Delta B \neq A \Delta C$ : therefore the cardinality $|\Delta \mathbf{F}|$ of $\Delta \mathbf{F}$ is always greater than or equal to the cardinality $|\mathbf{F}|$ of $\mathbf{F}$. Hence $m$ sets produce at least $m$ symmetric differencies (and at most $m(m-1) / 2+1$ : this upper bound is attained for every $m$, e.g. by $m$ pairwise disjoint sets).

Our first result, which will be proved in section 2, sharpens the above lower bound on $|\Delta \mathbf{F}|$ by showing that if $|\mathbf{F}|=m$ then $|\Delta \mathbf{F}| \geq 2^{\left\lceil\log _{2}(m)\right\rceil}$, i.e. that if $|\mathbf{F}|>2^{n}$ then $|\Delta \mathbf{F}| \geq 2^{n+1}$. Since a family of subsets of a set with $n+1$ elements can produce at most $2^{n+1}$ symmetric differences our lower bound on $|\Delta \mathbf{F}|$ is optimal. Our result in particular entails that if $|\Delta \mathbf{F}|=|\mathbf{F}|$, then $|\mathbf{F}|$ is a power of 2 and we will also prove that if $|\mathbf{F}|>2^{n}$ and $|\Delta \mathbf{F}|=2^{n+1}$, then there exists $\mathbf{F}^{\prime} \supseteq \mathbf{F}$ with $\left|\mathbf{F}^{\prime}\right|=2^{n+1}$ and $\left|\Delta \mathbf{F}^{\prime}\right|=\left|\mathbf{F}^{\prime}\right|$ (so that $\Delta \mathbf{F}^{\prime}=\Delta \mathbf{F}$ ).

This shows that to describe the set-theoretic structure of the families $\mathbf{F}$ with as few as possible symmetric differences, i.e. such that the lower bound on $|\Delta \mathbf{F}|$

Received by the editors September 27, 1996.

1991 Mathematics Subject Classification. Primary 04A03; Secondary 90D46.

This work has been supported by funds $40 \%$ and $60 \%$ MURST.

(C)1999 American Mathematical Society 
is attained, it suffices to describe the set-theoretic structure of the families $\mathbf{F}$ such that $|\Delta \mathbf{F}|=|\mathbf{F}|$. The main goal of sections 3 and 4 is to shed some light on this set-theoretic structure and this will be accomplished using two different approaches. In section 3 we will focus on the Venn diagram of the family, while in section 4 we will concentrate on the way the elements of the family can be distinguished by way of a tree construction.

\section{The LeAst nUmber of SYMMETRIC DIFFERENCES}

The power set $\operatorname{Pow}(X)$ of a set $X$ together with the operation $\Delta$ is a group with $\emptyset$ as the identity and the property that the inverse of any element is the element itself, namely a 2-group. Groups of this kind, also called Boolean groups, are wellknown and easily seen to be abelian, finite whenever finitely generated, and, in that case, to have order a power of 2 .

Proposition 2.1. If $\mathbf{F}$ is finite, then $\bar{\Delta} \mathbf{F}$ is a finite Boolean group with the operation $\Delta$, and $|\bar{\Delta} \mathbf{F}|=2^{n}$ for some $n$.

Finite Boolean groups provide the appropriate framework for the study of the operation $\Delta$ on finite families.

The following proposition collects a couple of elementary properties of cosets of subgroups of Boolean groups that will be useful in the sequel.

Proposition 2.2. Let $G$ be a Boolean group and $H$ be a subgroup of $G$.

1) Let $g, g^{\prime} \in G$. $g$ and $g^{\prime}$ are in the same coset of $H$ if and only if $g g^{\prime} \in H$.

2) If $g \in G$, then $H \cup(g H)$, i.e. the union of $H$ with the coset of $H$ containing $g$, is a subgroup of $G$.

Proof. 1) $g$ and $g^{\prime}$ are in the same coset of $H$ if and only if for some $h \in H g^{\prime}=g h$ if and only if $g g^{\prime} \in H$.

2) Every subset of $G$ is closed under inverses. By 1) the product of two elements of $g H$ belongs to $H$, as does the product of two elements of $H$, while the product of an element of $H$ with an element of $g H$ belongs to $g H$. Therefore $H \cup(g H)$ is closed under products.

The above facts about Boolean groups allow us to establish the lower bound on $|\Delta \mathbf{F}|$ stated in the introduction.

Theorem 2.3. For any $n$ and any family $\mathbf{F}$ with $|\mathbf{F}|>2^{n}$ we have $|\Delta \mathbf{F}| \geq 2^{n+1}$. Hence for every $m$ the least number of symmetric differences that $m$ sets can produce is $2^{\left\lceil\log _{2}(m)\right\rceil}$.

Proof. Let $\mathbf{G}=\bar{\Delta} \mathbf{F}$, which by Proposition 2.1 is a finite Boolean group with the operation $\Delta$. Moreover $|\mathbf{G}|=2^{n+1+k}$ for some $k \geq 0$. Let $h$ be maximal such that there exists a subgroup $\mathbf{H} \leq \mathbf{G}$ of order $2^{h}$ such that $\mathbf{H} \cap \Delta \mathbf{F}=\{\emptyset\}$.

We claim that $h \leq k$. In fact if $h>k$, then $\mathbf{H}$ has at most $2^{n}$ cosets and, since $|\mathbf{F}|>2^{n}$, one of them contains at least two distinct elements of $\mathbf{F}$ whose symmetric difference, by Proposition 2.2.1, would be a nonempty element of $\mathbf{H} \cap \Delta \mathbf{F}$.

A consequence of the claim is that $\mathbf{H}$ has at least $2^{n+1}$ cosets. If one of them has empty intersection with $\Delta \mathbf{F}$, then the union of this coset with $\mathbf{H}$ is a subgroup (by Proposition 2.2.2) of order $2^{h+1}$ which intersects $\Delta \mathbf{F}$ only in $\emptyset$, contradicting the maximality of $h$. Hence every coset of $\mathbf{H}$ contains at least one element of $\Delta \mathbf{F}$ and $|\Delta \mathbf{F}| \geq 2^{n+1}$. 
We now study the families for which the lower bound on the cardinality of the family of the symmetric differences is attained, i.e. families $\mathbf{F}$ such that for some $n,|\mathbf{F}|>2^{n}$ and $|\Delta \mathbf{F}|=2^{n+1}$.

Theorem 2.4. Let $\mathbf{F}$ be a nonempty finite family of sets. The following are equivalent:

i) $|\Delta \mathbf{F}|$ is the least number of symmetric differences $|\mathbf{F}|$ sets can produce;

ii) $|\mathbf{F}|>|\Delta \mathbf{F}| / 2$ and $\Delta \mathbf{F}$ is a group under $\Delta$;

iii) there exists $\mathbf{F}^{\prime} \supseteq \mathbf{F}$ such that $|\mathbf{F}|>\left|\mathbf{F}^{\prime}\right| / 2$ and $\left|\mathbf{F}^{\prime}\right|=\left|\Delta\left(\mathbf{F}^{\prime}\right)\right|$.

Proof. For $|\mathbf{F}|=1$ the theorem holds since i), ii), and iii) are all true. So let $n$ be such that $2^{n}<|\mathbf{F}| \leq 2^{n+1}$.

To prove that i) implies ii) notice that if $|\Delta \mathbf{F}|$ is the least number of symmetric differences $|\mathbf{F}|$ sets can produce then, since, as pointed out in the introduction, the lower bound in Theorem 2.3 is optimal, $|\Delta \mathbf{F}|=2^{n+1}$ and hence $|\mathbf{F}|>|\Delta \mathbf{F}| / 2$. Therefore we need only to show that $\Delta \mathbf{F}$ is closed under symmetric differences.

Let $\mathbf{G}=\bar{\Delta} \mathbf{F}$, so that by Proposition $2.1|\mathbf{G}|=2^{n+1+k}$ for some $k \geq 0$. If $k=0$, then $\mathbf{G}=\Delta \mathbf{F}$, and we are done; so we assume that $k \geq 1$.

We first show by induction on $i, 1 \leq i \leq k$, that for every $X \in \mathbf{G} \backslash(\Delta \mathbf{F})$ there exists a subgroup $\mathbf{H}$ of $\mathbf{G}$ of order $2^{i}$ such that $X \in \mathbf{H}$ and $\mathbf{H} \cap \Delta \mathbf{F}=\{\emptyset\}$. For $i=1$ simply take $\mathbf{H}=\{\emptyset, X\}$. Assume the property holds for $i<k$ and let $\mathbf{H}$ be of order $2^{i}$, with $X \in \mathbf{H}$ and $\mathbf{H} \cap \Delta \mathbf{F}=\{\emptyset\}$. $\mathbf{H}$ has $2^{n+1+k-i}>2^{n+1}$ cosets, and since $|\Delta \mathbf{F}|=2^{n+1}$ there are cosets of $\mathbf{H}$ which do not contain any element of $\Delta \mathbf{F}$. If $Y \Delta \mathbf{H}$ is one of them, then necessarily $Y \Delta \mathbf{H} \neq \mathbf{H}$, and then $\mathbf{H} \cup(Y \Delta \mathbf{H})$ is (by Proposition 2.2.2) a subgroup of $\mathbf{G}$ of order $2^{i+1}$ which contains $X$ and no element of $\Delta \mathbf{F}$ but $\emptyset$.

Suppose now that $A, B$ are such that $A \Delta B \notin \Delta \mathbf{F}$. Let $\mathbf{H}$ be a subgroup of $\mathbf{G}$ of order $2^{k}$ such that $A \Delta B \in \mathbf{H}$ and $\mathbf{H} \cap \Delta \mathbf{F}=\{\emptyset\}$. By Proposition 2.2.1 $A$ and $B$ belong to the same coset of $\mathbf{H}$, and by the proof of Theorem 2.3 if $|\Delta \mathbf{F}|=2^{n+1}$, then at most one of $A, B$ is in $\Delta \mathbf{F}$. Hence if $A, B \in \Delta \mathbf{F}$, then $A \Delta B \in \Delta \mathbf{F}$ and $\Delta \mathbf{F}$ is closed under $\Delta$.

To prove that ii) implies iii), assume ii) holds, fix any $A \in \mathbf{F}$, and let $\mathbf{F}^{\prime}=$ $A \Delta(\Delta \mathbf{F}) . \quad \mathbf{F}^{\prime} \supseteq \mathbf{F}$ holds because $A^{\prime}=A \Delta\left(A \Delta A^{\prime}\right)$ for every $A^{\prime} \in \mathbf{F}$, and hence $\Delta \mathbf{F} \subseteq \Delta\left(\mathbf{F}^{\prime}\right)$. Since $\Delta \mathbf{F}$ is a group $\mathbf{F}^{\prime}$ is a coset of $\Delta \mathbf{F}$ within $\bar{\Delta} \mathbf{F}$, so that $\left|\mathbf{F}^{\prime}\right|=$ $|\Delta \mathbf{F}|$, and $\Delta\left(\mathbf{F}^{\prime}\right) \subseteq \Delta \mathbf{F}$. Therefore $\Delta\left(\mathbf{F}^{\prime}\right)=\Delta \mathbf{F}$ and hence $\left|\Delta\left(\mathbf{F}^{\prime}\right)\right|=\left|\mathbf{F}^{\prime}\right|$.

iii) implies i) is immediate because iii) implies that $\left|\mathbf{F}^{\prime}\right|=\left|\Delta\left(\mathbf{F}^{\prime}\right)\right|=2^{n+1}$ and $\Delta \mathbf{F} \subseteq \Delta\left(\mathbf{F}^{\prime}\right)$. Hence $|\Delta \mathbf{F}|=2^{n+1}$ since by Theorem $2.3,|\Delta \mathbf{F}| \geq 2^{n+1}$.

Remark 2.5. The proof that ii) implies iii) of Theorem 2.4 shows that if $\mathbf{F}$ produces as few as possible symmetric differences, then either $|\bar{\Delta} \mathbf{F}|=2^{n+1}$ or $|\bar{\Delta} \mathbf{F}|=$ $2^{n+2}$. Nevertheless $|\bar{\Delta} \mathbf{F}|$ being small is not equivalent to $|\Delta \mathbf{F}|$ being least: $\mathbf{F}=$ $\{\{1\},\{2\},\{3\},\{4\},\{1,2,3,4\}\}$ is a family of $2^{2}+1$ sets such that $|\bar{\Delta} \mathbf{F}|=2^{4}$ but $|\Delta \mathbf{F}|=2^{3}+3$.

Remark 2.6. In ii) of Theorem 2.4 both conditions are necessary: any family of 4 sets producing 7 symmetric differences shows that $|\mathbf{F}|>|\Delta \mathbf{F}| / 2$ alone does not suffice to ensure that $|\Delta \mathbf{F}|$ is least, while $\mathbf{F}=\{\emptyset,\{1\},\{2\},\{3\},\{4\},\{1,2,3,4\}\}$ is a family of $2^{2}+2$ sets such that $\Delta \mathbf{F}$ is a group of order $2^{4}$ under $\Delta$, and hence shows that the closure of $\Delta \mathbf{F}$ under $\Delta$ does not suffice to ensure that $|\Delta \mathbf{F}|$ is least. 


\section{VENN DIAGRAMS}

Theorem 2.4 shows that if a family has few symmetric differences then it is contained in some $\mathbf{F}$ satisfying $|\Delta \mathbf{F}|=|\mathbf{F}|$. In this and in the next section we will study families satisfying $|\Delta \mathbf{F}|=|\mathbf{F}|$.

A special case is of course offered by the families $\mathbf{F}$ such that $\mathbf{F}=\Delta \mathbf{F}$, i.e. such that $(\mathbf{F}, \Delta)$ is a Boolean group; a condition which is clearly equivalent to $|\mathbf{F}|=|\Delta \mathbf{F}|$ and $\emptyset \in \mathbf{F}$. A typical example of a family $\mathbf{F}$ satisfying the equality $\mathbf{F}=\Delta \mathbf{F}$ is the power set $\operatorname{Pow}(X)$ of any given set $X$. On the other hand every finite Boolean group $G$ is easily seen to be isomorphic to a power set with the operation of symmetric difference. Indeed if $X$ is a minimal set of generators of $G$, every product of elements of $X$ equals the product of the elements of $X$ which actually occur in it an odd number of times, so that to every element of $G$ corresponds a unique subset of $X$. Furthermore the product of two elements in $G$ is exactly the product of the elements in the symmetric difference of their corresponding subsets of $X$.

Therefore from the algebraic point of view there are no solutions to the equation $\mathbf{F}=\Delta \mathbf{F}$ but the power sets. Moreover, since $|\mathbf{F}|=|\Delta \mathbf{F}|$ implies $\Delta \mathbf{F}=\Delta(\Delta \mathbf{F})$, from the same algebraic point of view the families $\mathbf{F}$ such that $|\mathbf{F}|=|\Delta \mathbf{F}|$ are again just the power sets. But this is far from being true as far as the set-theoretic structure of $\mathbf{F}$ is concerned.

In this section we explore the Venn diagrams that families satisfying $|\mathbf{F}|=|\Delta \mathbf{F}|$ can have. We will give first a characterization of the families satisfying $\mathbf{F}=\Delta \mathbf{F}$, and then show how to characterize the remaining solutions to the equation $|\mathbf{F}|=|\Delta \mathbf{F}|$ by making use of the operation, to be defined below, of forming a Venn variant of a family of sets.

Definition 3.1. $\quad$ - Let $\mathcal{G}_{n}$ be the family of sets of cardinality $n$ of the form

$$
\left\{\left\{s_{1}\right\} \cup A_{1}, \ldots,\left\{s_{n}\right\} \cup A_{n}\right\}
$$

where the $s_{i}$ are all distinct and $\left\{s_{1}, \ldots, s_{n}\right\} \cap\left(A_{1} \cup \ldots \cup A_{n}\right)=\emptyset$.

- Let $\mathcal{D}_{n}=\left\{\bar{\Delta} \mathbf{G} \mid \mathbf{G} \in \mathcal{G}_{n}\right\}$.

We will use the following set-theoretic notion, which has been introduced in [1].

Definition 3.2. $S$ is a differentiating set for the family $\mathbf{F}$ if for every $A, B \in \mathbf{F}$ with $A \neq B$ we have $A \cap S \neq B \cap S$, i.e. $(A \Delta B) \cap S \neq \emptyset$. $S$ is a minimal differentiating set for $\mathbf{F}$ if it is a differentiating set and for every $s \in S, S \backslash\{s\}$ is not a differentiating set for $\mathbf{F}$.

In [1] it is shown that if $|\mathbf{F}|=m$, then $\mathbf{F}$ has a differentiating set $S$ of cardinality $m-1$.

Theorem 3.3. $\quad$ 1) If $\mathbf{F} \in \mathcal{D}_{n}$, then $|\mathbf{F}|=2^{n}$ and $\mathbf{F}=\Delta \mathbf{F}$;

2) If $\mathbf{F}=\Delta \mathbf{F}$, then for every minimal differentiating set $S$ for $\mathbf{F}$ the map $A \mapsto$ $A \cap S$ is a bijection between $\mathbf{F}$ and $\operatorname{Pow}(S)$;

3) $\mathbf{F}=\Delta \mathbf{F}$ if and only if for some $n, \mathbf{F} \in \mathcal{D}_{n}$.

Proof. 1) Let $\mathbf{G}=\left\{\left\{s_{1}\right\} \cup A_{1}, \ldots,\left\{s_{n}\right\} \cup A_{n}\right\} \in \mathcal{G}_{n}$ and $\mathbf{F}=\bar{\Delta} \mathbf{G} \in \mathcal{D}_{n}$. Then $\emptyset \in \mathbf{F}$ and obviously $\mathbf{F}=\Delta \mathbf{F}$. Furthermore the fact that the $s_{i}$ are all distinct and the condition $\left\{s_{1}, \ldots, s_{n}\right\} \cap\left(A_{1} \cup \ldots \cup A_{n}\right)=\emptyset$ ensure that no element of $\mathbf{G}$ can be generated from the others by means of $\Delta$. Hence they are a minimal set of generators of $\mathbf{F}$, which therefore has cardinality $2^{n}$. 
2) Assume that $\mathbf{F}=\Delta \mathbf{F}$ so that $\mathbf{F}$ is closed under $\Delta$. In general for $\mathbf{F}$ closed under $\Delta$, from the identity $A \Delta(A \Delta(B \Delta C))=B \Delta C$, it follows that for every $A \in \mathbf{F}$ we have $\Delta \mathbf{F}=A \Delta \mathbf{F}$. Let $S$ be a minimal differentiating set for $\mathbf{F}$. For $X \in \Delta \mathbf{F}$ let $\pi(X)=X \cap S$. Since $S$ is a differentiating set for $\mathbf{F}, \pi$ is one-to-one. In fact if $(A \Delta B) \cap S=(A \Delta C) \cap S$ then $(B \Delta C) \cap S=\emptyset$; therefore if for $A, B, C \in \mathbf{F}$ with $B \neq C$ we had $\pi(A \Delta B)=\pi(A \Delta C), S$ would fail to be a differentiating set for $\mathbf{F}$. Furthermore, from the identity $(X \cap S) \Delta(Y \cap S)=(X \Delta Y) \cap S$ and the closure of $\Delta \mathbf{F}$ under $\Delta$, it follows that the range of $\pi$ is closed under $\Delta$ as well. Finally, by the minimality of $S$, for every $s \in S$ there exist $X \in \Delta \mathbf{F}$ such that $\pi(X)=X \cap S=\{s\}$. Thus every subset of $S$, being the symmetric difference of the singletons of its elements, is in the range of $\pi . \pi$ is therefore a bijection between $\Delta \mathbf{F}$ and $\operatorname{Pow}(S)$.

3) The "if" part follows immediately from 1). For the "only if" part let $S=$ $\left\{s_{1}, \ldots, s_{n}\right\}$ be a minimal differentiating set for $\mathbf{F}$ as in the proof of 2). If $X_{1}=\left\{s_{1}\right\} \cup A_{1}, \ldots, X_{n}=\left\{s_{n}\right\} \cup A_{n}$ are the elements of $\Delta \mathbf{F}$ that intersect $S$ in singletons, we have

$$
\Delta \mathbf{F}=\bar{\Delta}\left\{\left\{s_{1}\right\} \cup A_{1}, \ldots,\left\{s_{n}\right\} \cup A_{n}\right\}
$$

and $\left\{s_{1}, \ldots, s_{n}\right\} \cap\left(A_{1} \cup \ldots \cup A_{n}\right)=\emptyset$. Thus $\mathbf{F} \in \mathcal{D}_{n}$.

Remark 3.4. $\mathcal{D}_{n}$ does not exhaust all the families $\mathbf{F}$ with $2^{n}$ elements such that $|\mathbf{F}|=|\Delta \mathbf{F}|$; for example the family $\mathbf{F}=\{\{1\},\{2\}\}$ is such that $\Delta \mathbf{F}=\{\emptyset,\{1,2\}\}$, so that $|\mathbf{F}|=|\Delta \mathbf{F}|$, although $\mathbf{F} \neq \Delta \mathbf{F}$.

We now prove that if $|\Delta \mathbf{F}|$ is minimal then the size of any minimal differentiating set for $\mathbf{F}$ is minimal.

Proposition 3.5. If $|\mathbf{F}|>2^{n},|\Delta \mathbf{F}|=2^{n+1}$ and $S$ is a minimal differentiating set for $\mathbf{F}$, then $|S|=n+1$.

Proof. If $S$ is a minimal differentiating set for $\mathbf{F}$, then $|S| \geq n+1$. For every $s \in S$, $\{s\} \in S \cap \Delta \mathbf{F}$. By Theorem 2.4 $\Delta \mathbf{F}$ is closed under $\Delta$, and therefore $S \cap \Delta \mathbf{F}$ is closed under $\Delta$. It follows that $\operatorname{Pow}(S)=S \cap \Delta \mathbf{F}$ and thus $|S| \leq n+1$.

Remark 3.6. The converse of Proposition 3.5 is false even in the special case $|\mathbf{F}|=$ $2^{n+1}$ : $\mathbf{F}$ can be such that $|\mathbf{F}|=2^{n+1}$ and have only minimal differentiating sets of the smallest possible size, i.e. $n+1$, but fail to satisfy $|\Delta \mathbf{F}|=2^{n+1}$. An example is provided by $\mathbf{F}=\{\emptyset,\{1\},\{2\},\{1,2,3\}\}$ which is a family of $2^{2}$ sets generating $2^{2}+3$ symmetric differences, although its only minimal differentiating set is $\{1,2\}$.

Starting with families in $\mathcal{D}_{n}$, new families satisfying the equality $|\mathbf{F}|=|\Delta \mathbf{F}|$ can be obtained by the operation of making what we call Venn variants.

Definition 3.7. - Let $V(\mathbf{F})$ denote the Venn diagram of $\mathbf{F}$, namely the partition induced on $\bigcup \mathbf{F}$ by the equivalence relation $\sim_{\mathbf{F}}$ defined by

$$
x \sim_{\mathbf{F}} y \text { if and only if } \forall A \in \mathbf{F}(x \in A \longleftrightarrow y \in A) .
$$

- For $v \in V(\mathbf{F})$ we let

$$
\mathbf{F}_{v}=\{A \backslash v \mid A \in \mathbf{F} \wedge v \subseteq A\} \cup\{A \cup v \mid A \in \mathbf{F} \wedge A \cap v=\emptyset\}
$$

and call $\mathbf{F}_{v}$ the Venn variant of $\mathbf{F}$ determined by $v$.

The following proposition is immediate from the definition. 
Proposition 3.8. For all $v \in V(\mathbf{F}),\left|\mathbf{F}_{v}\right|=|\mathbf{F}|$ and $\Delta\left(\mathbf{F}_{v}\right)=\Delta \mathbf{F}$, i.e. a Venn variant of $\mathbf{F}$ has the same cardinality and produces the same collection of symmetric differences as $\mathbf{F}$.

Definition 3.9. $\mathcal{V}_{n}$ is the least family which contains $\mathcal{D}_{n}$ and is closed under formation of Venn variants, namely

- if $\mathbf{F} \in \mathcal{D}_{n}$, then $\mathbf{F} \in \mathcal{V}_{n}$;

- if $\mathbf{F} \in \mathcal{V}_{n}$ and $v \in V(\mathbf{F})$, then $\mathbf{F}_{v} \in \mathcal{V}_{n}$.

By Theorem 3.3 and iterated application of Proposition 3.8 we obtain:

Proposition 3.10. If $\mathbf{F} \in \mathcal{V}_{n}$, then $|\mathbf{F}|=|\Delta \mathbf{F}|=2^{n}$.

The analysis we will now carry out will show that not even $\mathcal{V}_{n}$ exhausts the families of cardinality $2^{n}$ which have only $2^{n}$ symmetric differencies.

Definition 3.11. For $v, v^{\prime} \in V(\mathbf{F})$ we say that $v$ is opposite to $v^{\prime}$ in $\mathbf{F}$ if

$$
\{A \in \mathbf{F} \mid v \subseteq A\}=\left\{A \in \mathbf{F} \mid A \cap v^{\prime}=\emptyset\right\}
$$

or, equivalently, if

$$
\{A \in \mathbf{F} \mid v \subseteq A\}=\mathbf{F} \backslash\left\{A \in \mathbf{F} \mid v^{\prime} \subseteq A\right\} .
$$

Proposition 3.12. Let $\mathbf{F}$ be a family and $v, v^{\prime}, v^{\prime \prime} \in V(\mathbf{F})$ :

1) $v$ is not opposite to $v$ in $\mathbf{F}$;

2) if $v$ is opposite to $v^{\prime}$ in $\mathbf{F}$, then $v^{\prime}$ is opposite to $v$ in $\mathbf{F}$;

3) if $v^{\prime}$ and $v^{\prime \prime}$ are opposite to $v$ in $\mathbf{F}$, then $v^{\prime}=v^{\prime \prime}$;

4) if no element of $V(\mathbf{F})$ is opposite to $v$ in $\mathbf{F}$, then $V\left(\mathbf{F}_{v}\right)=V(\mathbf{F})$, so that for every $u \in V(\mathbf{F}) \mathbf{F}_{v u}$ is defined; furthermore $\mathbf{F}_{v v}=\mathbf{F}$;

5) if $v$ and $v^{\prime}$ are opposite in $\mathbf{F}$, then

$$
V\left(\mathbf{F}_{v}\right)=\left(V(\mathbf{F}) \backslash\left\{v, v^{\prime}\right\}\right) \cup\left\{v \cup v^{\prime}\right\},
$$

so that for every $u \in V(\mathbf{F}) \backslash\left\{v, v^{\prime}\right\} \mathbf{F}_{v u}$ is defined; furthermore $\mathbf{F}_{v}=\mathbf{F}_{v^{\prime}}$;

6) if $v \in V(\mathbf{F})$, then either $v$ (if $v$ has no opposite in $\mathbf{F}$ ) or $v \cup v^{\prime}$ (if $v$ and $v^{\prime}$ are opposite in $\mathbf{F}$ ) has no opposite in $\mathbf{F}_{v}$;

7) for any $v \in V(\mathbf{F})$, if $v^{\prime}$ and $v^{\prime \prime}$ are not opposite in $\mathbf{F}$ then $v^{\prime}$ and $v^{\prime \prime}$ are not opposite in $\mathbf{F}_{v}$.

Proof. 1) and 2) are immediate.

3) Let $A \in \mathbf{F} . v^{\prime} \subseteq A$ is equivalent (since $v$ and $v^{\prime}$ are opposite) to $v \cap A=\emptyset$ which is equivalent (since $v$ and $v^{\prime \prime}$ are opposite) to $v^{\prime \prime} \subseteq A$. Hence

$$
\left\{A \in \mathbf{F} \mid v^{\prime} \subseteq A\right\}=\left\{A \in \mathbf{F} \mid v^{\prime \prime} \subseteq A\right\},
$$

which entails $v^{\prime}=v^{\prime \prime}$.

4) It suffices to show that $\sim_{\mathbf{F}}$ and $\sim_{\mathbf{F}_{v}}$ are the same equivalence relation. Let $x, y \in \bigcup \mathbf{F}$. If $x \sim_{\mathbf{F}} y$, then either $x, y \in v$ or $x, y \notin v$, from which it follows immediately that $x \sim_{\mathbf{F}_{v}} y$. Conversely let us assume that $x \nsim_{\mathbf{F}} y$ so that at most one of $x, y$ is in $v$. If $x, y \notin v$ let $A \in \mathbf{F}$ be such that $x \in A$ and $y \notin A$; then one of $A \backslash v$ and $A \cup v$ is in $\mathbf{F}_{v}$ and witnesses that $x \nsim_{\mathbf{F}_{v}} y$. Now suppose $x \in v$, and hence $y \notin v$. Since $v$ has no opposite element in $\mathbf{F}$, there is $B \in \mathbf{F}$ such that either $x, y \in B$ or $x, y \notin B$. In the former case $B \backslash v$ contains $y$ but does not contain $x$; in the latter case $B \cup v$ contains $x$ but does not contain $y$. In both cases we have that $x \nsim_{\mathbf{F}_{v}} y$.

$\mathbf{F}_{v v}=\mathbf{F}$ follows immediately from the definitions. 
5) The proof of 4) shows that if $u \in V(\mathbf{F}), u \neq v$ and $u \neq v^{\prime}$, then $u$ is a $\sim_{\mathbf{F}_{v}}$ equivalence class, namely an element of $V\left(\mathbf{F}_{v}\right)$. On the other hand if $x \in v$ and $y \in v^{\prime}$, then $x \sim_{\mathbf{F}_{v}} y$ so that $v \cup v^{\prime}$ replaces both $v$ and $v^{\prime}$ in $V\left(\mathbf{F}_{v}\right)$.

6) Let $\bar{v}$ be either $v$ (if $v$ has no opposite in $\mathbf{F}$ ) or $v \cup v^{\prime}$ (if $v$ and $v^{\prime}$ are opposite in $\mathbf{F})$. Suppose $u \in V\left(\mathbf{F}_{v}\right)$ is opposite to $\bar{v}$ in $\mathbf{F}_{v}$. By 1) $u \neq \bar{v}$ and hence by 4) or 5) $u \in V(\mathbf{F})$. Since $u \neq v$ let $A \in \mathbf{F}$ be such that either $u \subseteq A$ and $v \nsubseteq A$ or $u \nsubseteq A$ and $v \subseteq A$. In the former case $A \cup v \in \mathbf{F}_{v}$ and contains both $u$ and $\bar{v}$; in the latter case $A \backslash v \in \mathbf{F}_{v}$ and contains neither $u$ nor $\bar{v}$. In both cases $u$ and $\bar{v}$ are not opposite in $\mathbf{F}_{v}$.

7) If $v$ is either $v^{\prime}$ or $v^{\prime \prime}$, then the conclusion follows immediately from 6). Otherwise $v^{\prime}, v^{\prime \prime} \in V(\mathbf{F})$ and, since $v^{\prime}$ and $v^{\prime \prime}$ are not opposite in $\mathbf{F}$, there exists $A \in \mathbf{F}$ such that either $v^{\prime} \subseteq A$ and $v^{\prime \prime} \subseteq A$ or $v^{\prime} \cap A=\emptyset$ and $v^{\prime \prime} \cap A=\emptyset$. Then either $A \cup v$, if $v \cap A=\emptyset$, or $A \backslash v$, if $v \subseteq A$, witnesses that $v^{\prime}$ and $v^{\prime \prime}$ are not opposite in $\mathbf{F}_{v}$.

As an immediate consequence we have the following:

Corollary 3.13. If $V(\mathbf{F})$ has no pair of opposite elements, then for every $v \in$ $V(\mathbf{F}), V\left(\mathbf{F}_{v}\right)$ has no pair of opposite elements.

The following proposition relates the absence of opposite elements in the Venn diagram of a family with the Venn diagram of the family of its symmetric differences.

Proposition 3.14. $V(\mathbf{F})$ has no pair of opposite elements if and only if $V(\mathbf{F})=$ $V(\Delta \mathbf{F})$.

Proof. Let $x \sim_{\mathbf{F}}^{-} y$ stand for $\forall A \in \mathbf{F}(x \in A \longleftrightarrow y \notin A)$. As it is easy to check $x \sim_{\Delta \mathbf{F}} y$ if and only if either $x \sim_{\mathbf{F}} y$ or $x \sim_{\mathbf{F}}^{-} y$, so that $V(\Delta \mathbf{F})$ is the partition induced on $\bigcup \mathbf{F}$ by the equivalence relation $x \sim_{\mathbf{F}} y \vee x \sim_{\mathbf{F}}^{-} y$. If $V(\mathbf{F})$ has no pair of opposite elements, $\sim_{\mathbf{F}}^{-}$is the empty relation and $\sim_{\Delta \mathbf{F}}$ coincides with $\sim_{\mathbf{F}}$ so that $V(\mathbf{F})=V(\Delta \mathbf{F})$. Conversely if $V(\mathbf{F})=V(\Delta \mathbf{F})$, then $\sim_{\mathbf{F}}=\sim_{\Delta \mathbf{F}}$ and $\sim_{\mathbf{F}}^{-}$must be empty, which entails that in $V(\mathbf{F})$ there are no pairs of opposite elements.

Proposition 3.15. If $\mathbf{F} \in \mathcal{V}_{n}$, then $V(\mathbf{F})$ has no pair of opposite elements.

Proof. If $\mathbf{F} \in \mathcal{D}_{n}$, then $\mathbf{F}=\Delta \mathbf{F}$; thus $V(\mathbf{F})=V(\Delta \mathbf{F})$ so that by the previous proposition $V(\mathbf{F})$ has no pair of opposite elements. Since the families in $\mathcal{V}_{n}$ are obtained from those in $\mathcal{D}_{n}$ by iterating the operation of Venn variant, by Corollary 3.13 their Venn diagrams have no pairs of opposite elements.

Remark 3.16. If $\mathbf{F}=\{\{1\},\{2\},\{1,3\},\{2,3\}\}$, then $\Delta \mathbf{F}=\{\emptyset,\{1,2\},\{3\},\{1,2,3\}\}$ so that $|\mathbf{F}|=|\Delta \mathbf{F}|$. However $\{1\}$ and $\{2\}$ are elements of $V(\mathbf{F})$ which are opposite in $\mathbf{F}$, so that $\mathbf{F} \notin \mathcal{V}_{2}$ by Proposition 3.15.

The following proposition shows that the families in $\mathcal{V}_{n}$ are precisely those which have the least possible number of symmetric differences and, at the same time, have no pair of opposite elements in their Venn diagram.

\section{Proposition 3.17.}

$$
\mathcal{V}_{n}=\left\{\mathbf{F}|| \mathbf{F}|=| \Delta \mathbf{F} \mid=2^{n} \wedge V(\mathbf{F}) \text { has no pair of opposite elements }\right\} .
$$

Proof. Propositions 3.10 and 3.15 show that $\mathcal{V}_{n}$ is included in the set on the right hand side of the equality. 
To prove the reverse inclusion assume that $|\mathbf{F}|=|\Delta \mathbf{F}|=2^{n}$ and $V(\mathbf{F})$ has no pair of opposite elements. Let $S$ be a minimal differentiating set for $\mathbf{F}$. Since $S$ is a differentiating set for $\mathbf{F}$, the map $\lambda: \mathbf{F} \rightarrow \operatorname{Pow}(S)$ defined by $\lambda(A)=A \cap S$ is oneto-one. Since $|\mathbf{F}|=|\Delta \mathbf{F}|, \Delta \mathbf{F}=\Delta(\Delta \mathbf{F})$, and, by Theorem 3.3.2, $|\Delta \mathbf{F}|=|\operatorname{Pow}(S)|$. Therefore $\lambda$ is actually a bijection. Thus there exists a unique $A_{0} \in \mathbf{F}$ such that $A_{0} \cap S=\emptyset$. Let $v_{1}, \ldots, v_{k} \in V(\mathbf{F})$ be such that $A_{0}=v_{1} \cup \ldots \cup v_{k}$. Since $V(\mathbf{F})$ has no pair of opposite elements $\mathbf{G}=\mathbf{F}_{v_{1} \ldots v_{k}}$ is well defined. Clearly $\emptyset \in \mathbf{G}$ and by Proposition $3.8|\mathbf{G}|=|\Delta \mathbf{G}|$. As noticed earlier these conditions entail $\mathbf{G}=\Delta \mathbf{G}$. Thus by Theorem 3.3 we have that $\mathbf{G} \in \mathcal{D}_{n}$. Finally, since by Proposition 3.12.4

$$
\begin{aligned}
\mathbf{F} & =\mathbf{F}_{v_{1} \ldots v_{k} v_{k} v_{k-1} \ldots v_{1}} \\
& =\mathbf{G}_{v_{k} \ldots v_{1}},
\end{aligned}
$$

we have that $\mathbf{F} \in \mathcal{V}_{n}$.

By Proposition 3.12.6 and 7, eliminating pairs of opposite elements is simply a matter of iterating the operation of making Venn variants.

Definition 3.18. If $\left(v_{1}, v_{1}^{\prime}\right), \ldots,\left(v_{k}, v_{k}^{\prime}\right)$ are all the pairs of opposite elements in $\mathbf{F}$, we let

$$
\mathbf{F}^{*}=\mathbf{F}_{v_{1} \ldots v_{k}} .
$$

Note that $\mathbf{F}^{*}$ depends neither on the order in which $v_{1}, \ldots, v_{k}$ are taken (since, in general, $\mathbf{F}_{v u}=\mathbf{F}_{u v}$ as long as both Venn variants are legal), nor on which element of the pair $\left(v_{i}, v_{i}^{\prime}\right)$ is used to make the Venn variant (by Proposition 3.12.5). As an immediate consequence of Proposition 3.12.6 and 7 we have the following:

Proposition 3.19. $V\left(\mathbf{F}^{*}\right)$ has no pair of opposite elements.

We can now state our characterization of the finite families satisfying $|\mathbf{F}|=|\Delta \mathbf{F}|$.

Theorem 3.20. $|\mathbf{F}|=|\Delta \mathbf{F}|=2^{n}$ if and only if $\mathbf{F}^{*} \in \mathcal{V}_{n}$.

Proof. Since by Proposition $3.8|\mathbf{F}|=\left|\mathbf{F}^{*}\right|$ and $\Delta \mathbf{F}=\Delta\left(\mathbf{F}^{*}\right)$, the if part follows from Proposition 3.10, while the only if part follows from Propositions 3.19 and 3.17 .

\section{TREES DESCRIBING A FAMILY}

In this section we provide a different characterization of the familes $\mathbf{F}$ satisfying $|\mathbf{F}|=|\Delta \mathbf{F}|$. This characterization is based on the analysis of how elements of $(\bigcup \mathbf{F}) \backslash(\bigcap \mathbf{F})$ discriminate between the sets in $\mathbf{F}$.

Definition 4.1. Given a family $\mathbf{F}$ and an element $x \in(\bigcup \mathbf{F}) \backslash(\bigcap \mathbf{F})$, we let

$$
\mathbf{F}_{x}=\{A \in \mathbf{F} \mid x \in A\} \quad \text { and } \quad \mathbf{F}_{\bar{x}}=\{A \in \mathbf{F} \mid x \notin A\} .
$$

We begin with some simple facts that will turn out to be useful.

Proposition 4.2. For any $x \in(\bigcup \mathbf{F}) \backslash(\bigcap \mathbf{F})$ we have

1) $\Delta \mathbf{F}=\left(\Delta \mathbf{F}_{x}\right) \cup\left(\Delta \mathbf{F}_{\bar{x}}\right) \cup\left(\mathbf{F}_{x} \Delta \mathbf{F}_{\bar{x}}\right)$;

2) $\left[\left(\Delta \mathbf{F}_{x}\right) \cup\left(\Delta \mathbf{F}_{\bar{x}}\right)\right] \cap\left(\mathbf{F}_{x} \Delta \mathbf{F}_{\bar{x}}\right)=\emptyset$;

3) $|\Delta \mathbf{F}| \geq 2 \max \left(\left|\mathbf{F}_{x}\right|,\left|\mathbf{F}_{\bar{x}}\right|\right)$.

Proof. 1) is immediate. 2) follows from the fact that for every $A \in\left(\Delta \mathbf{F}_{x}\right) \cup\left(\Delta \mathbf{F}_{\bar{x}}\right)$ we have $x \notin A$ while for every $B \in \mathbf{F}_{x} \Delta \mathbf{F}_{\bar{x}}$ we have $x \in B$. 3) is a consequence of 1) and 2) together with $|\mathbf{F} \Delta \mathbf{G}| \geq \max (|\mathbf{F}|,|\mathbf{G}|)$. 
Proposition 4.3. If $|\mathbf{F}|=|\Delta \mathbf{F}|$ and $x \in(\bigcup \mathbf{F}) \backslash(\bigcap \mathbf{F})$, then $\left|\Delta \mathbf{F}_{x}\right|=\left|\mathbf{F}_{x}\right|=$ $\left|\Delta \mathbf{F}_{\bar{x}}\right|=\left|\mathbf{F}_{\bar{x}}\right|=|\mathbf{F}| / 2$ and $\Delta \mathbf{F}_{x}=\Delta \mathbf{F}_{\bar{x}}$.

Proof. $\left|\mathbf{F}_{x}\right|=\left|\mathbf{F}_{\bar{x}}\right|=|\mathbf{F}| / 2$ follows immediately from the hypothesis and Proposition 4.2.3. Since $\left|\mathbf{F}_{x} \Delta \mathbf{F}_{\bar{x}}\right| \geq|\mathbf{F}| / 2$ by Proposition 4.2 .2 we must have $\Delta \mathbf{F}_{x}=\Delta \mathbf{F}_{\bar{x}}$ and $\left|\Delta \mathbf{F}_{x}\right|=\left|\Delta \mathbf{F}_{\bar{x}}\right|=|\mathbf{F}| / 2$.

Definition 4.4. A tree describing $\mathbf{F}$ is a binary tree satisfying the following properties:

1. the nodes are pairwise different subsets of $\mathbf{F}$,

2. the $\operatorname{root}$ is $\mathbf{F}$,

3. the leaves are singleton subsets of $\mathbf{F}$,

4. the children of any internal node $\mathbf{G} \subseteq \mathbf{F}$ are $\mathbf{G}_{x}$ and $\mathbf{G}_{\bar{x}}$ for some $x \in$ $(\cup \mathbf{G}) \backslash(\bigcap \mathbf{G})$.

The height $h(\mathbf{F})$ of a family $\mathbf{F}$ is the height of the highest tree describing $\mathbf{F}$.

The next theorem gives another characterization of the finite families satisfying $|\mathbf{F}|=|\Delta \mathbf{F}|$.

Theorem 4.5. $|\mathbf{F}|=|\Delta \mathbf{F}|$ if and only if $h(\mathbf{F})=\log _{2}(|\mathbf{F}|)$.

Proof. By induction on $|\mathbf{F}|$.

If $|\mathbf{F}|=1$ the only tree describing $\mathbf{F}$ consists only of the root, and hence the theorem holds.

If $|\mathbf{F}|>1$ consider any tree describing $\mathbf{F}$ and let $x \in(\bigcup \mathbf{F}) \backslash(\bigcap \mathbf{F})$ be such that $\mathbf{F}_{x}$ and $\mathbf{F}_{\bar{x}}$ are the children of the root. The subtrees lying above these nodes describe respectively $\mathbf{F}_{x}$ and $\mathbf{F}_{\bar{x}}$; if $|\mathbf{F}|=|\Delta \mathbf{F}|$ by Proposition 4.3 and the inductive hypothesis we have that their heights are $\log _{2}(|\mathbf{F}|)-1$ and hence that the original tree has height $\log _{2}(|\mathbf{F}|)$.

If $h(\mathbf{F})=\log _{2}(|\mathbf{F}|)$, then both $\mathbf{F}_{x}$ and $\mathbf{F}_{\bar{x}}$ have height less than or equal to $\log _{2}(|\mathbf{F}|)-1$. Hence their size is bounded by $2^{\log _{2}(|\mathbf{F}|)-1}=|\mathbf{F}| / 2$. From this we can conclude that $\left|\mathbf{F}_{x}\right|=\left|\mathbf{F}_{\bar{x}}\right|=|\mathbf{F}| / 2$ and that $h\left(\mathbf{F}_{x}\right)=h\left(\mathbf{F}_{\bar{x}}\right)=\log _{2}(|\mathbf{F}|)-1$. By inductive hypothesis we have that $\left|\mathbf{F}_{x}\right|=\left|\Delta \mathbf{F}_{x}\right|=\left|\mathbf{F}_{\bar{x}}\right|=\left|\Delta \mathbf{F}_{\bar{x}}\right|=|\mathbf{F}| / 2$.

We begin by showing that $|\mathbf{F}|=|\Delta \mathbf{F}|$ holds if $|\mathbf{F}|=4$. Let $\mathbf{F}=\{A, B, C, D\}$ and notice that $C \Delta D=A \Delta B$; otherwise $A \Delta B \Delta C \Delta D \neq \emptyset$ and there exists $x \in$ $(\bigcup \mathbf{F}) \backslash(\bigcap \mathbf{F})$ belonging to either exactly one or exactly three elements of $\mathbf{F}$ : in both cases we could construct a tree describing $\mathbf{F}$ of height 3 . Therefore we have also $B \Delta C=A \Delta D$ and $B \Delta D=A \Delta C$, so that $\Delta \mathbf{F}=\{A \Delta A, A \Delta B, A \Delta C, A \Delta D\}$ and hence $|\mathbf{F}|=|\Delta \mathbf{F}|$.

We now turn to the general case. Let $\alpha$ be a sequence indexing a node in a tree describing $\mathbf{F}$, and let $\beta$ be a sequence obtained from $\alpha$ replacing zero or more characters $x$ by $\bar{x}$, and zero or more characters $\bar{x}$ by $x$. For example: $\alpha=x \bar{y} z \bar{w}$ and $\beta=\bar{x} \bar{y} z w$.

Let also denote by $\bar{\gamma}$ the sequence obtained from $\gamma$ replacing each $x$ by $\bar{x}$ and each $\bar{x}$ by $x$.

The following equality will be proved by induction on $|\mathbf{F}|$ :

$$
\mathbf{F}_{\alpha} \Delta \mathbf{F}_{\beta}=\mathbf{F}_{\bar{\alpha}} \Delta \mathbf{F}_{\bar{\beta}} .
$$

The base case is immediate.

The inductive step is proved by a further induction on $\log _{2}(|\mathbf{F}|)-|\alpha|$. 
The base case corresponds to the case in which $|\alpha|=\log _{2}(|\mathbf{F}|)$ and is the most complex. In this case, let $\mathbf{F}_{\alpha}=\{A\}, \mathbf{F}_{\beta}=\{B\}, \mathbf{F}_{\bar{\alpha}}=\left\{A^{\prime}\right\}$, and $\mathbf{F}_{\bar{\beta}}=\left\{B^{\prime}\right\}$; we must prove that $A \Delta B=A^{\prime} \Delta B^{\prime}$.

If $\alpha=\bar{\beta}$ the result is obvious. Otherwise let $x$ be an element occurring both in $\alpha$ and in $\beta$ (the argument for the case where only elements of the form $\bar{x}$ occur both in $\alpha$ and in $\beta$ is analogous). We can assume without loss of generality that $\alpha=x \alpha_{1}$ and $\beta=x \beta_{1}$. By inductive hypothesis on the cardinality of the family,

$$
\mathbf{F}_{\alpha} \Delta \mathbf{F}_{\beta}=\mathbf{F}_{x \alpha_{1}} \Delta \mathbf{F}_{x \beta_{1}}=\mathbf{F}_{x \overline{\alpha_{1}}} \Delta \mathbf{F}_{x \overline{\beta_{1}}} .
$$

Moreover

$$
\begin{aligned}
& \mathbf{F}_{\bar{\alpha}}=\mathbf{F}_{\bar{x} \overline{\alpha_{1}}}=\left\{A^{\prime}\right\} \Longrightarrow \mathbf{F}_{\overline{\alpha_{1}}}=\left\{A^{\prime}, A^{\prime \prime}\right\} \text { with } x \in A^{\prime \prime}, \\
& \mathbf{F}_{\bar{\beta}}=\mathbf{F}_{\bar{x} \overline{\beta_{1}}}=\left\{B^{\prime}\right\} \Longrightarrow \mathbf{F}_{\overline{\beta_{1}}}=\left\{B^{\prime}, B^{\prime \prime}\right\} \text { with } x \in B^{\prime \prime} .
\end{aligned}
$$

From this it follows that $\mathbf{F}_{x \overline{\alpha_{1}}}=\left\{A^{\prime \prime}\right\}$ and $\mathbf{F}_{x \overline{\beta_{1}}}=\left\{B^{\prime \prime}\right\}$. Hence $\mathbf{F}_{\alpha} \Delta \mathbf{F}_{\beta}=$ $\mathbf{F}_{x \overline{\alpha_{1}}} \Delta \mathbf{F}_{x \overline{\beta_{1}}}$ implies that $A \Delta B=A^{\prime \prime} \Delta B^{\prime \prime}$ and it suffices to prove $A^{\prime \prime} \Delta B^{\prime \prime}=A^{\prime} \Delta B^{\prime}$.

To this end consider the family $\mathbf{G}=\left\{A^{\prime}, B^{\prime}, A^{\prime \prime}, B^{\prime \prime}\right\}$. We show that $h(\mathbf{G})=$ $\log _{2}(|\mathbf{G}|)=2$ : let $\mathbf{H}$ be the first common ancestor of $\mathbf{F}_{\overline{\alpha_{1}}}=\left\{A^{\prime}, A^{\prime \prime}\right\}$ and $\mathbf{F}_{\overline{\beta_{1}}}=$ $\left\{B^{\prime}, B^{\prime \prime}\right\}$, and let $\mathbf{H}_{y}$ and $\mathbf{H}_{\bar{y}}$ be the children of $\mathbf{H}$. Since $y$ discriminates between $\left\{A^{\prime}, A^{\prime \prime}\right\}$ and $\left\{B^{\prime}, B^{\prime \prime}\right\}$, either $y$ appears in $\overline{\alpha_{1}}$ or it appears in $\overline{\beta_{1}}$. Assuming, without loss of generality, that the former is the case, we have that for some $\xi$, $\mathbf{F}_{\bar{\alpha}}=\mathbf{F}_{\xi \bar{x} y}$ and hence $\mathbf{F}_{\xi}=\left\{A^{\prime}, A^{\prime \prime}, B^{\prime}, B^{\prime \prime}\right\}=\mathbf{G}$. If we had a tree describing $\mathbf{G}$ of height greater than two, we could graft such a tree in place of $\mathbf{F}_{\xi}$ in a tree describing $\mathbf{F}$ and produce a tree describing $\mathbf{F}$ of height greater than $\log _{2}(|\mathbf{F}|)$, contradicting the hypothesis.

Hence $h(\mathbf{G})=2$ and by the case $|\mathbf{F}|=4$ considered above, $A^{\prime \prime} \Delta B^{\prime \prime}=A^{\prime} \Delta B^{\prime}$, which concludes the base case.

For the inductive step pick $x \in \bigcup\left(\mathbf{F}_{\alpha} \Delta \mathbf{F}_{\beta}\right) \backslash \bigcap\left(\mathbf{F}_{\alpha} \Delta \mathbf{F}_{\beta}\right)$ and notice that

$$
\begin{aligned}
\mathbf{F}_{\alpha} \Delta \mathbf{F}_{\beta} & =\left(\mathbf{F}_{\alpha} \Delta \mathbf{F}_{\beta}\right)_{x} \cup\left(\mathbf{F}_{\alpha} \Delta \mathbf{F}_{\beta}\right)_{\bar{x}} \\
& =\left[\left(\mathbf{F}_{\alpha x} \Delta \mathbf{F}_{\beta \bar{x}}\right) \cup\left(\mathbf{F}_{\alpha \bar{x}} \Delta \mathbf{F}_{\beta x}\right)\right] \cup\left[\left(\mathbf{F}_{\alpha x} \Delta \mathbf{F}_{\beta x}\right) \cup\left(\mathbf{F}_{\alpha \bar{x}} \Delta \mathbf{F}_{\beta \bar{x}}\right)\right] \\
& =\left[\left(\mathbf{F}_{\bar{\alpha} \bar{x}} \Delta \mathbf{F}_{\bar{\beta} x}\right) \cup\left(\mathbf{F}_{\bar{\alpha} x} \Delta \mathbf{F}_{\bar{\beta} \bar{x}}\right)\right] \cup\left[\left(\mathbf{F}_{\bar{\alpha} \bar{x}} \Delta \mathbf{F}_{\bar{\beta} \bar{x}}\right) \cup\left(\mathbf{F}_{\bar{\alpha} x} \Delta \mathbf{F}_{\bar{\beta} x}\right)\right] \\
& =\left(\mathbf{F}_{\bar{\alpha}} \Delta \mathbf{F}_{\bar{\beta}}\right)_{x} \cup\left(\mathbf{F}_{\bar{\alpha}} \Delta \mathbf{F}_{\bar{\beta}}\right)_{\bar{x}} \\
& =\mathbf{F}_{\bar{\alpha}} \Delta \mathbf{F}_{\bar{\beta}}
\end{aligned}
$$

where the third equality has been obtained by induction hypothesis.

If $\alpha=\beta=x$, the equality we just proved shows that $\Delta \mathbf{F}_{x}=\Delta \mathbf{F}_{\bar{x}}$. Since we already have $\left|\Delta \mathbf{F}_{x}\right|=|\mathbf{F}| / 2$, Proposition 4.2.1 entails that our thesis $|\mathbf{F}|=|\Delta(\mathbf{F})|$ will follow from

$$
\left|\mathbf{F}_{x} \Delta \mathbf{F}_{\bar{x}}\right|=|\mathbf{F}| / 2 .
$$

Since $\left|\Delta \mathbf{F}_{x}\right|=\left|\mathbf{F}_{x}\right|$ and $\left|\Delta \mathbf{F}_{\bar{x}}\right|=\left|\mathbf{F}_{\bar{x}}\right|$ for any $A \in \mathbf{F}_{x}$ and $B \in \mathbf{F}_{\bar{x}}$, we have $\Delta \mathbf{F}_{x}=\left\{A \Delta A^{\prime} \mid A^{\prime} \in \mathbf{F}_{x}\right\}$ and $\Delta \mathbf{F}_{\bar{x}}=\left\{B \Delta B^{\prime} \mid B^{\prime} \in \mathbf{F}_{\bar{x}}\right\}$. Fix $B \in \mathbf{F}_{\bar{x}}$ and consider the function $\varphi: \mathbf{F}_{x} \rightarrow \mathbf{F}_{x} \Delta \mathbf{F}_{\bar{x}}$ defined by $\varphi\left(A^{\prime}\right)=A^{\prime} \Delta B$.

Clearly $\varphi$ is injective. $\varphi$ is also surjective: for any $A^{\prime} \Delta B^{\prime} \in \mathbf{F}_{x} \Delta \mathbf{F}_{\bar{x}}$, since $B \Delta B^{\prime} \in \Delta \mathbf{F}_{\bar{x}}=\Delta \mathbf{F}_{x}$ there exists $A^{\prime \prime} \in \mathbf{F}_{x}$ such that $B \Delta B^{\prime}=A^{\prime} \Delta A^{\prime \prime}$. Hence

$$
A^{\prime} \Delta B^{\prime}=A^{\prime \prime} \Delta B=\varphi\left(A^{\prime \prime}\right) .
$$

Hence $\varphi$ is a bijection between $\mathbf{F}_{x}$ and $\mathbf{F}_{x} \Delta \mathbf{F}_{\bar{x}}$ : we have $\left|\mathbf{F}_{x} \Delta \mathbf{F}_{\bar{x}}\right|=\left|\mathbf{F}_{x}\right|=|\mathbf{F}| / 2$ and the proof is complete. 
Remark 4.6. The generalization of Theorem 4.5 stating that for any family $\mathbf{F}$, $|\Delta \mathbf{F}|=2^{\left\lceil\log _{2}(|\mathbf{F}|)\right\rceil}$ if and only if $h(\mathbf{F})=\left\lceil\log _{2}(|\mathbf{F}|)\right\rceil$ is false. The "only if" direction follows easily by Theorems 2.4 and 4.5 , but the "if" direction does not hold. A counterexample is provided by $\mathbf{F}=\{\emptyset,\{1,2\},\{1,3\},\{1,4\},\{2,3,4\}\}$, which is a family of $2^{2}+1$ sets generating $2^{3}+3$ symmetric differences, although $h(\mathbf{F})=\left\lceil\log _{2}(|\mathbf{F}|)\right\rceil=3$. Notice also that this family has only minimal differentiating sets of size 3 (see Remark 3.6).

The result in this section can be interpreted in the following, playful, way. A family of sets $\mathbf{F}$ is given and players $I$ and $I I$ have full knowledge of $\mathbf{F}$. Player $I$ picks $X \in \mathbf{F}$. Player $I I$ has to discover $X$, by asking, one after another, questions of the form "does $a$ belong to $X$ ?". Player $I$ has to give correct yes/no answers. Player $I I$ asks only questions whose answer he/she cannot recover from his/her knowledge of $\mathbf{F}$ and from the answers to the previous questions. When $\mathbf{F}$ is finite, player $I I$ will always discover $X$ after asking at most $|\mathbf{F}|-1$ questions. In general, the number of questions $I I$ has to ask depends on $X$ as well as on the sequence of the questions asked. For $\mathbf{F}$ finite, Theorem 4.5 says that the number of questions player $I I$ has to ask depends neither on $X$ nor on the sequence of the questions asked if and only if $|\mathbf{F}|=|\Delta \mathbf{F}|$. The results in Section 3 then give a way of constructing plays of that sort.

\section{ACKNOWLEDGMENT}

We have benefited from helpful discussions with prof. K.P.S.B. Rao.

\section{REFERENCES}

[1] F. Parlamento, A. Policriti, and K.P.S.B. Rao, Witnessing Differences Without Redundancies, Proceedings of the American Mathematical Society, 125 (1997), no. 2, 587-594. MR 97d:04003

(A. Marcone) Dipartimento di Matematica, Università di Torino, via Carlo Alberto 10, 10123 TORINo, ITALY

E-mail address: marcone@dm.unito.it

Current address: Dipartimento di Matematica e Informatica, Università di Udine, viale delle Scienze, 33100 Udine, Italy

E-mail address: marcone@dimi.uniud.it

(F. Parlamento and A. Policriti) Dipartimento di Matematica e Informatica, Università di Udine, viale delle Scienze, 33100 Udine, Italy

E-mail address: parlamen@dimi.uniud.it

E-mail address: policrit@dimi.uniud.it 